\title{
Sustainable Wireless Broadband Access to the Future Internet - The EARTH Project*
}

\author{
Dietrich Zeller ${ }^{1}$, Magnus Olsson ${ }^{2}$, Oliver Blume ${ }^{1}$, Albrecht Fehske ${ }^{3}$, \\ Dieter Ferling ${ }^{1}$, William Tomaselli ${ }^{4}$, and István Gódor ${ }^{2}$ \\ ${ }^{1}$ Alcatel-Lucent \\ ${ }^{2}$ Ericsson \\ ${ }^{3}$ Technical University of Dresden \\ ${ }^{4}$ Telecom Italia
}

\begin{abstract}
In a world of continuous growth of economies and global population eco-sustainability is of outmost relevance. Especially, mobile broadband networks are facing an exponential growing traffic volume and so the sustainability of these networks comes into focus. The recently completed European funded Seventh Framework Programme (FP7) project EARTH has studied the impact of traffic growth on mobile broadband network energy consumption and carbon footprint, pioneering this field. This chapter summarizes the key insights of EARTH on questions like "How does the exploding traffic impact the sustainability?", "How can energy efficiency be rated and predicted?", "What are the key solutions to improve the energy efficiency and how to efficiently integrate such solutions?" The results are representing the foundation of the maturing scientific engineering discipline of Energy Efficient Wireless Access, targeting the standardisation in IETF and 3GPP, strongly influencing academic research trends, and will soon be reflected in products and deployments of the European telecommunications industry.
\end{abstract}

\section{$1 \quad$ Introduction}

This chapter gives an overview of the FP7 project EARTH contributions to a sustainable wireless broadband access to the Future Internet. Hence, it summarizes the results of common work of the EARTH consortium obtained during the project duration from January 2010 until July 2012 [1].

The Europe 2020 strategy [2] of the European Union aims towards Smart, Sustainable and Inclusive Growth for Europe. In all these areas ICT (Information and Communication Technologies) are broadly considered as the lever to enable this growth. For instance, the Smart2020 report [3] stated that ICT can lead to emission reductions by $15 \%$ in 2020 compared to the emissions resulting from "business as usual".

A key part of the ICT infrastructure is represented by the Internet evolving to the Future Internet. The access to the Future Internet will be dominated by wireless devices. Already now most European citizens witness how much the Internet and mobile access to the Internet transforms their ways to live. But this is just the beginning

\footnotetext{
* Invied Paper.
}

A. Galis and A. Gavras (Eds.): FIA 2013, LNCS 7858, pp. 249-271, 2013.

(C) The Author(s). This article is published with open access at link.springer.com 
towards the envisaged society by Europe 2020. All these developments results in an explosive traffic growth, illustrated in Fig.1, which challenges the sustainability of networks.

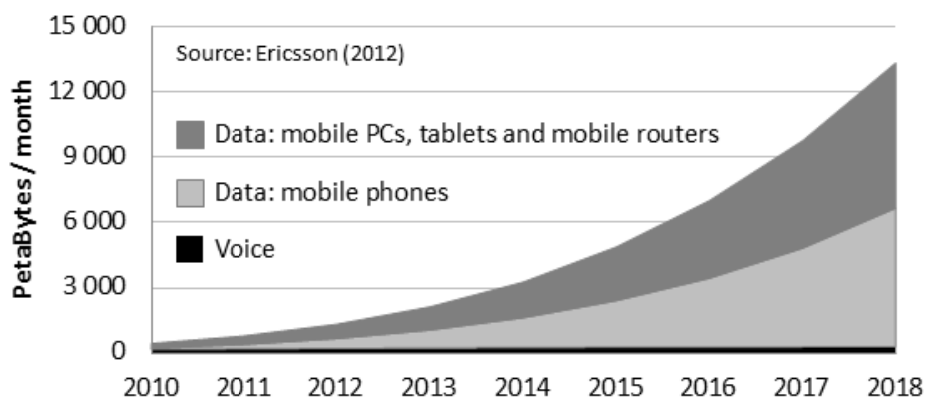

Fig. 1. Evolution of Mobile Data Traffic per Month up to 2018

Obviously, this growth is accompanied by an increased energy consumption of mobile networks, with a corresponding increase in the carbon footprint of the networks. Therefore telecommunication networks and in particular mobile networks have the tendency to increasingly contribute to global energy consumption. And this increase comes at a period where energy prices are extremely volatile and have resumed their upward trend. Rising energy costs have led to a situation where they are a significant part of network operation costs. In fact, operator's OPEX figures indicate that their energy costs are nowadays comparable to their personnel costs for network operations. All this clearly shows that the sustainability of network growth is at risk. But a sustainable growth of networks is the prerequisite that they can play their key role in enabling Smart, Sustainable and Inclusive Growth. So it is pivotal that methods are found ensuring the sustainable growth.

This context was the starting point for the EARTH project [1], a concerted effort with 15 partners from industry, SME and academia addressing improvements of the energy efficiency of mobile communication infrastructure. The project was guided by the ambitious overall goal to derive solutions that together in an Integrated Solution decrease the network energy consumption by $50 \%$ without degrading quality of service. The saving is understood as the saving a network deployment having the EARTH solutions in place would have compared to a deployment without these solutions. Both deployments are assumed to be subjected to identical traffic demands.

With this EARTH was addressing the call of EU Commissioner Viviane Reding on ICT industry for intensified efforts to reduce its carbon footprint by $20 \%$ as early as 2015 for tackling climate change [4].

In the subsequent sections we first present an analysis of the socio-economic impact of the traffic growth and the EARTH targeted savings. Then we present the EARTH methodology to evaluate the impact of different solutions on the network energy consumption. After presenting solutions on the radio and network level the EARTH approach to integrate solutions efficiently into Integrated Solutions is 
presented. The validation of the theoretical results by tests of prototype implementations under realistic conditions is described before the chapter is concluded with a summary.

\section{Socio-Economic Impact Analysis}

EARTH has developed a methodology that allows to accurately quantify the overall global carbon footprint of mobile communications in the period 2007 - 2020 considering the complete network lifecycle. We identify the energy consumption of global RAN (radio access network) operation as a main contributor and further investigate the potential impact of EARTH technologies on RAN energy consumption in several scenarios.

The model is based on detailed life cycle analysis of network equipment as well as models and data on development of mobile traffic volumes, number of base stations, and subscribers globally. We consider all generations of cellular mobile networks including all end-user equipment accessing the networks, all business activities of the operators running the networks, and the use of fixed network resources as a result of data traffic generated by mobile network users. Estimates on the number of mobile subscriptions, traffic volumes, and network infrastructure are based on projections from analysts Gartner and ABI Research and extrapolated for the period 2015 to 2020 as part of the EARTH project. For more details we refer to [5].

Over the last decade, the energy consumption of RAN equipment has already decreased by about $8 \%$ per year. This average annual improvement can be attributed to the technology scaling of semiconductors, as well as to improved radio access technologies. Consequently, this $8 \%$ per year improvement scenario is taken as a reference throughout the study and referred to as "continuous improvements".

\subsection{Global Carbon Footprint of Mobile Communications}

- According to the projection (see Fig.2), the overall carbon footprint of mobile communications increases almost linearly until 2020 with annual increase of 11 Mto $\mathrm{CO}_{2} \mathrm{e}$, equivalent to the annual emissions of 2.5 million EU households. The emissions in 2020 amount to more than $235 \mathrm{MtoCO}_{2} \mathrm{e}$, corresponding to more than one third of the present annual emissions of the entire United Kingdom. Relative to 2007, the overall carbon footprint will increase by a factor of 2 until 2014 and a factor of 2.7 until 2020. In the event that only minor efficiency improvements of base station sites and end-user equipment occur, the footprint could even increase more than threefold. In contrast, the footprint of the ICT sector as a whole is expected to increase by a factor of only 1.7 during the same 13-year period.

- While RAN operation was by far the largest contributor in 2007, mobile device manufacturing will develop an equal share in the overall carbon footprint in 2020. The reason for this is that smartphones and laptops represent an actively increasing fraction of the devices accessing the network - a trend driven by the demand for advanced wireless services and applications, especially video. Compared to regular 
phones, smartphones and laptops have carbon footprints almost two times and ten times higher, respectively.

- From 2007 to 2020, the annual data traffic volume per mobile subscription is expected to increase substantially from 0.3 GBytes/year up to 100 GBytes/year. The rather moderate linear increase in global footprint compared to the vast exponential increase in traffic volume is made possible by 1) strong increase in network capacity through small cells, 2) increasing spectral efficiency and bandwidths in future mobile standards, and 3) already existing unused capacity.

- An estimated number of 100 million femto cells in 2020 will consume about 4.4 $\mathrm{TWh} /$ year, which is less than $5 \%$ of that estimated for the global RAN operation in 2020. We infer that the total carbon emissions due to femto cells are comparably small and become significant only if their number approaches the order of 1 billion or more globally.

- The carbon footprint due to manufacturing and operation of M2M communication devices will be small, even for a vast number of existing devices in 2020. Here, only the actual modem part is allocated to be a part of the mobile network.

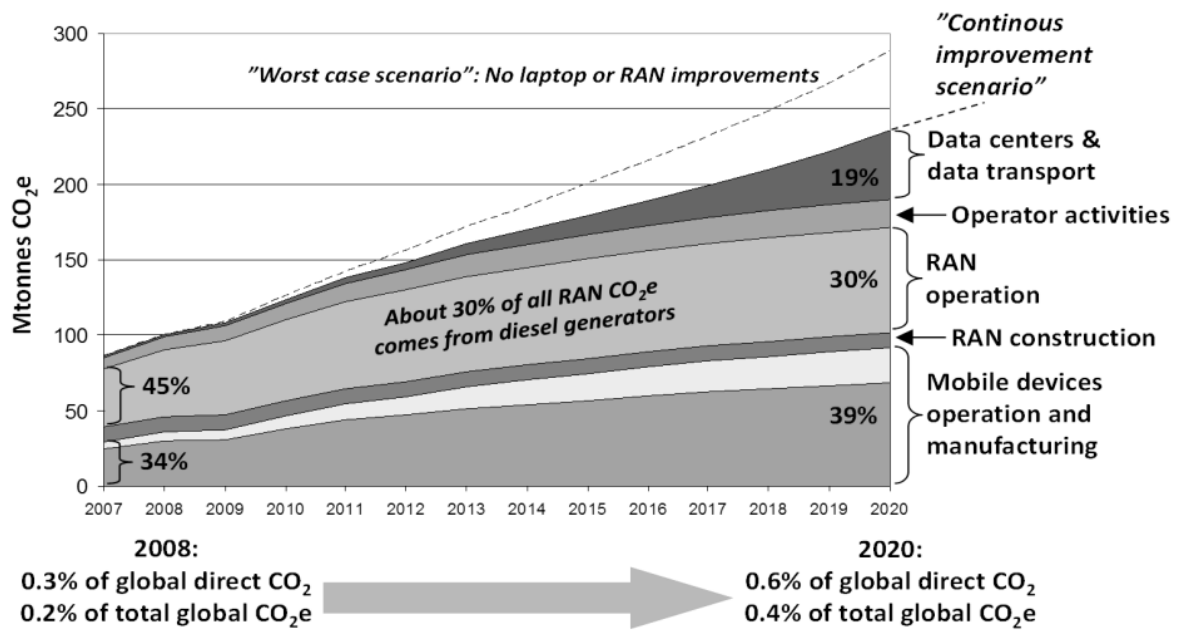

Fig. 2. Global carbon footprint of mobile communications in $\mathrm{CO}_{2} \mathrm{e}$ projected until 2020

\subsection{RAN Energy Consumption and the Potential Impact of EARTH Technologies}

As discussed above, a major source of $\mathrm{CO}_{2} \mathrm{e}$ from the network part of mobile communications is from the electrical power used for operation of the base stations (BS) in the RAN. Focusing on this topic we derived the following observations (see Fig.3):

- In the reference case of $8 \%$ improvements in efficiency per year, the RAN energy increases by about $28 \%$ in 2020 compared to 2012 (Scenario "Improvements $8 \%$ p.a." in Fig.3). 
- Assuming all base stations deployed in the period 2013-2020 use 50\% less electricity (the target of EARTH) RAN operation will increase only slightly compared to its 2012 value - despite the anticipated growth in traffic demand (Scenario "New technologies" in Fig.3). The 50\% reduction must be seen as per-site-average due to the combined effects of improved hardware as well as better use of the equipment through improved radio resource management, smarter deployment, and other improvements of the EARTH integrated solution.

- A significant reduction of RAN operation energy for 2020 is possible if innovations are also implemented in already installed base stations, e.g. through software updates, and site modernization (Scenario "Large swap of equipment" in Fig.3). Here, we assume a progressive swap-out of almost 40 percent of globally installed equipment, where old sites are replaced by state-of-the-art equipment during the period 2013 to 2020 .

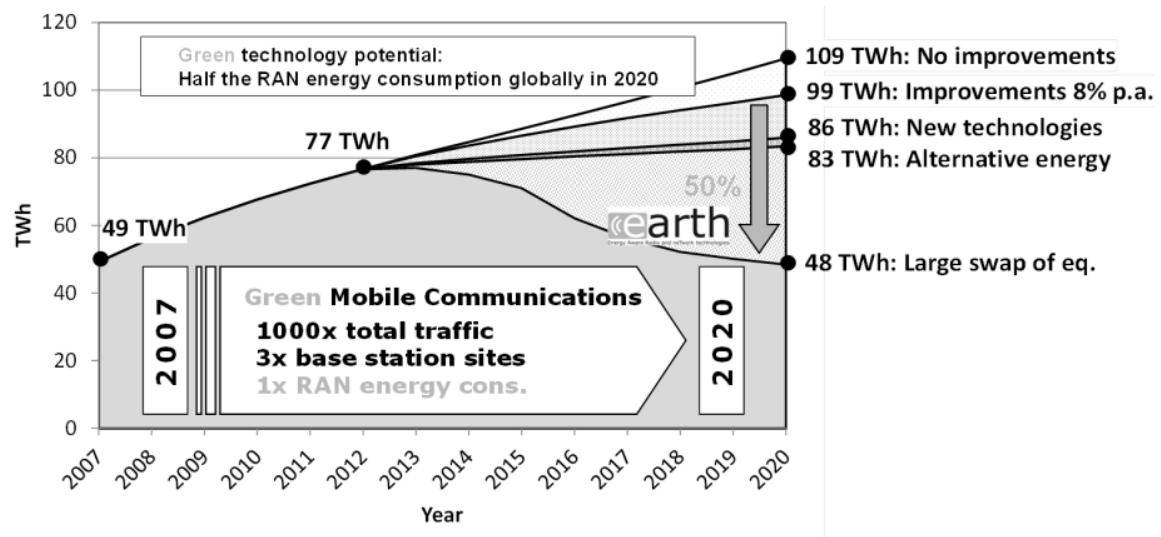

Fig. 3. Global RAN electricity consumption in TWh per year projected until 2020 for different scenarios of technology adoption

In summary, our analysis demonstrates that network operators should focus on savings in RAN operation. A 50\% reduction in energy consumption per site yields significant energy savings under realistic assumptions on technology adoption. It shows that with the saving target of EARTH it would be possible to keep the total power consumption of RANs flat after 2012, despite the expected exponential traffic growth. Application of EARTH solutions to the BSs deployed before 2012 would even enable to revert the growth in RAN power consumption experienced between 2007 and 2012.

\section{Energy Efficiency Evaluation Framework $\left(\mathbf{E}^{3} \mathbf{F}\right)$}

When the project started, no widely accepted methodology existed in the mobile industry and academia to quantify the energy efficiency of a wireless network. Metrics and measurement specification for the energy efficiency of individual base stations had been specified by the standard body ETSI [6]. However, for a large-scale 
network, like a national deployment of an operator this is much more difficult to achieve. Nevertheless, GSMA has recently provided a framework for the assessment and comparison of energy efficiency of large deployed networks in the field [7]. The limitation of this benchmarking service is in the assessment of saving potentials. Which parts or functions of the network are the main consumers and how much impact would certain improvements, e.g. in hardware or network management, achieve? EARTH has undertaken the effort to fill the methodology gap for predicting gains in efficiency in theory and in simulations and to build best practice advise for characteristic network scenarios.

The EARTH Energy Efficiency Evaluation Framework $\left(E^{3} F\right)$ [8] takes as starting point the well-known radio network assessment methodology of the Third Generation Partnership Project (3GPP), which is focused on small-scale scenarios and provides results in terms of system throughput, quality of service (QoS) metrics, and fairness in terms of cell edge user throughput. EARTH has extended this to the energy efficiency of large area networks with diverse environments ranging from rural areas to densely populated cities. The most important addends are a sophisticated power model for various base station types, as well as a large-scale long-term traffic model that allows for a holistic energy efficiency analysis over large geographical areas and extended periods of time (typically 24 hours instead of seconds). The EARTH E ${ }^{3} \mathrm{~F}$ is illustrated in Fig.4 and comprises the following steps:

1. Small-scale, short-term evaluations are conducted for each deployment environment (dense urban, urban, suburban and rural) and for a representative set of traffic loads, which captures the range between the minimum and the maximum load observed in a certain deployment environment.

2. The system level evaluations provide energy consumption and other performance metrics (e.g. throughput, QoS) for each small-scale deployment environment and a certain traffic load.

3. Given the daily traffic profile of a certain deployment environment, the power consumption over a day is generated by weighted summing of the short-term evaluations.

4. Finally, the mix of deployment environments that quantify the area covered by cities, suburbs, highways and villages, yield the global set of the large-scale system energy consumption.

The EARTH $E^{3} F$ has found application in the work of ETSI TC EE on defining how to compute "Network Energy Efficiency" [9]. Also the $\mathrm{E}^{3} \mathrm{~F}$ is already well adopted by the scientific community (e.g. in the Green Touch initiative [10]).

\subsection{Small-Scale Short-Term System-Level Evaluations}

System level simulations, requiring a lot of computation resources, are not feasible on the global scale and can only be executed for individual scenarios ("snapshots"). Further, studying snapshots, the $\mathrm{E}^{3} \mathrm{~F}$ enables to identify the most critical contributions to the global network and to study the best improvement strategy for each scenario individually. 


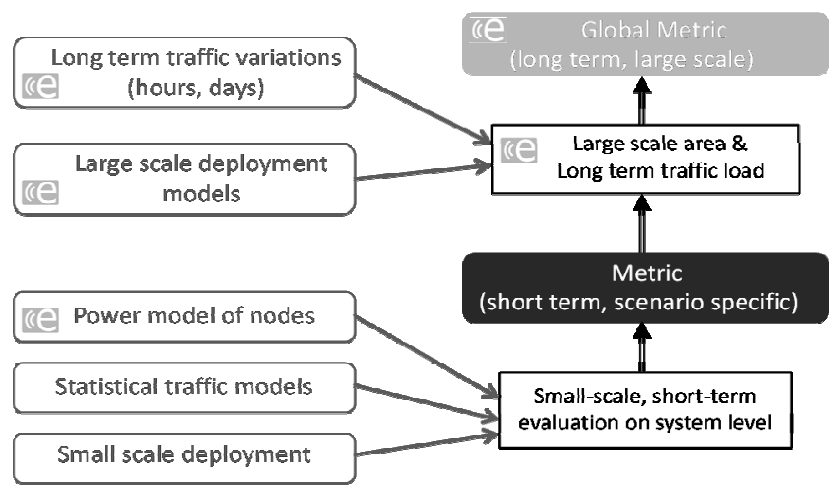

Fig. 4. EARTH Energy Efficiency Evaluation Framework $\left(\mathrm{E}^{3} \mathrm{~F}\right)$

Statistical traffic models (e.g. FTP file download or voice over IP calls), as well as specific small-scale deployment scenarios (e.g. urban macrocell consisting of 57 hexagonal cells with uniformly distributed users), constitute small-scale short-term system-level evaluations (bottom block in Fig.4). These evaluations are carried out by a system-level simulation platform, augmented by a model capturing the BS power consumption.

\subsection{Power Model}

The BS power model constitutes the interface between the component and system levels, which allows the quantification of how energy savings on specific components enhance the energy efficiency at the network level. The characteristics of the components largely depend on the BS type, due to constraints in output power, size, and cost. These heterogeneous characteristics mandate a power model that is tailored to a specific BS type.

Fig.5 shows power functions for typical macro and pico base stations (BS). A BS consists of multiple transceivers (TRXs), each of which serves one transmit antenna element. A TRX comprises a power amplifier (PA), a radio frequency (RF) smallsignal TRX module, a baseband (BB) engine including a receiver (uplink) and transmitter (downlink) section, a DC-DC power supply, an active cooling (CO) system, and a power supply (PS) for connection to the electrical power grid. In the model the power consumption of the various TRX parts are analysed and combined to the total input power, as illustrated in Fig.5.

Examination of the BS power consumption as a function of its load reveals that mainly the PA scales with the BS load. However, this scaling over signal load largely depends on the BS type. While the power consumption $\mathrm{P}_{\text {in }}$ is load-dependent for macro BSs, the load dependency of pico BSs is negligible. The reason is that for low power nodes the PA accounts for less than $30 \%$ of the overall power consumption, whereas for macro BSs the PA power consumption amounts to 55-60\% of the total. Other components hardly scale with the load in contemporary implementations. 


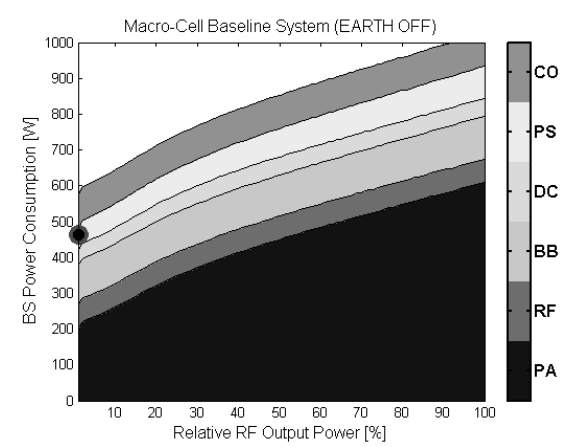

(a) Macro base station

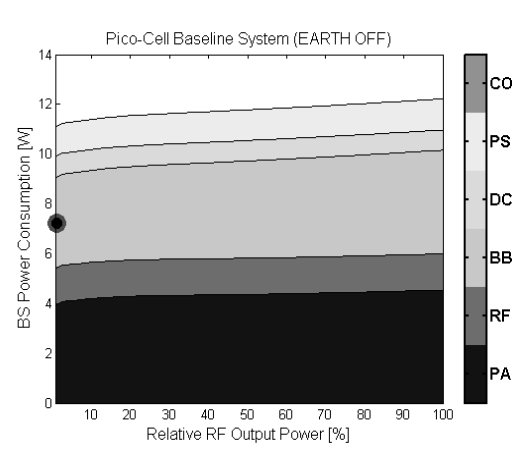

(b) Pico base station

Fig. 5. Base station power model (State of the Art)

The power model has been published both in high level [8] and in detail [11] and has found widespread use in the literature of energy efficiency calculations. Furthermore it has been introduced into 3GPP standardization [12].

\subsection{Traffic Model}

The $\mathrm{E}^{3} \mathrm{~F}$ methodology captures the traffic demand by modelling the subscriber density, the data rates of their devices and their daily activity patterns. The traffic model is based on the UMTS Forum's mobile traffic forecast [13].

The model considers different deployment types: dense urban, urban, rural and suburban areas. The basic parameters of the model are depicted in Fig.6. In Europe, these constitute about half the total area and their population densities are such that highly populated areas only cover a few percent of Europe. More than half of the area is so sparsely populated that there basically is no economic case for LTE deployment. These areas are neglected in the model.

The expected traffic volume per subscriber is increasingly dominated by mobile broadband subscribers. Essentially, the traffic model represents a range equivalent of continuous heavy usage of high-definition video streaming with display resolutions of $1280 \times 720$ pixels (720p HD) and "light" usage of intensive web browsing. Due to the diminishing share of voice traffic, voice subscribers are not included in our model.

The resulting traffic demand in dense urban areas at peak hours yields between 83 $\mathrm{Mb} / \mathrm{s} / \mathrm{km}^{2}$ for $20 \%$ heavy users and $276 \mathrm{Mb} / \mathrm{s} / \mathrm{km}^{2}$ for $100 \%$ heavy users. For urban and rural deployments the traffic demand is computed using up to 7 times lower activity levels and up to 30 times lower population density. Note that usually several operators share the traffic so that the range of traffic demand scenarios to be used in the small scale system level simulations spans $0.1-100 \mathrm{Mbps} / \mathrm{km}^{2}$.

Fig.6(c) shows potential energy saving resulting from traffic adaptive energy consumption, i.e., serving lower traffic should require lower energy. 


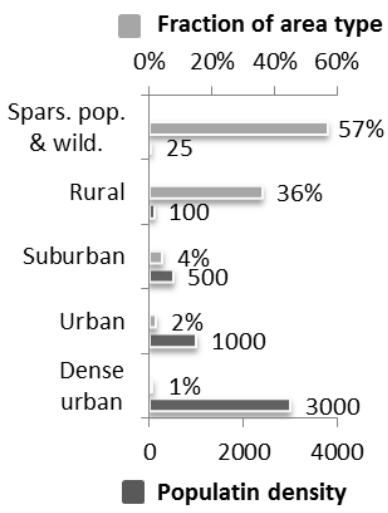

(a) Deployment model

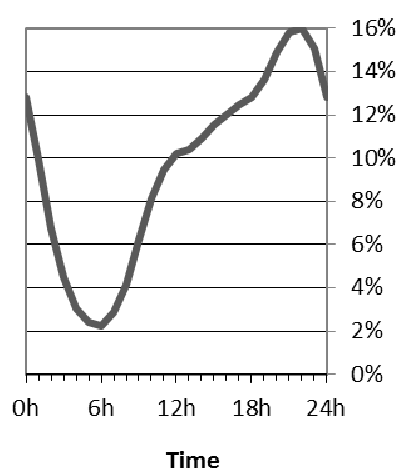

(b) Daily variation of traffic

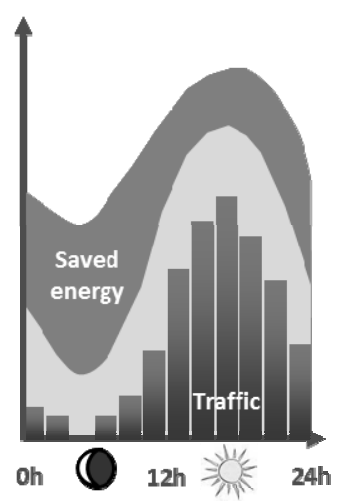

(c) Potential saving

Fig. 6. E3F traffic model parameters: population density, fraction of area types, daily variation of traffic and potential savings resulting from traffic adaptive energy consumption

\subsection{Metrics}

Different energy intensity metrics provide dissimilar perspectives on the energy consumption. For expressing the energy saving in access networks the power per area unit metric, in $\left[\mathrm{W} / \mathrm{m}^{2}\right]$, is the primary choice by EARTH. The energy per bit metric, in [J/bit], and the power per subscriber metric, in [W/sub], make up useful complementary metrics. Whereas the power per area unit metric focuses on the total energy consumption and the saving potential at a given traffic scenario, the energy per bit metric provides a figure on the bit delivery energy efficiency and is useful rather for comparing scenarios with long-term exponential traffic growth. The power per subscriber metric is easily observed in real networks, and offers stability over long time periods, which makes it a suitable candidate for energy consumption measurement in real networks.

\subsection{Application of $\mathbf{E}^{3} \mathbf{F}$ : Where Is the Energy Saving Potential?}

One of the first activities in the project was to carry out a situation analysis, i.e., to assess the energy efficiency of a typical mobile broadband network and thereby identify the most important improvement areas to focus on. The purpose of this analysis was to answer questions such as where and when energy is consumed in a typical radio access network. For this, the $E^{3} \mathrm{~F}$ was used to analyse the power consumption of a European LTE reference network, operating at $2 \mathrm{GHz}$.

The obtained results reveal that the energy intensity in urban areas is nearly 10 times higher than for rural areas, due to the higher density of base stations. However, the aggregation of $E^{3} F$ reveals a roughly equal impact of rural and urban areas on the total energy consumption because rural areas are by far dominating area wise, 
c.f. Fig.6(a). This indicates that both urban and rural areas need to be considered, and are equally important to consider, when improving energy efficiency of a network.

The analysis also showed that the network operates at relatively low load levels. In the studied network, less than $10 \%$ of the subframes are utilized for transmission of user data. Still, due to local temporal and geographical variations certain parts of the network must serve a large number of simultaneously active users during shorter time periods. The analysis further revealed that for current network design and operation the power consumption is only weakly dependent of the traffic load. This is a clear indication that the no and low load situations are where the largest energy saving potential is. Furthermore, traditionally radio access research both in the academy and the industry have been focused on the challenge to achieve as high data rates as possible for a given maximum transmission power. Therefore current technologies can be considered to already be fairly energy efficient during transmission. This further supports the conclusion that the largest unexplored energy saving potential is to be found in low and no load situations.

The potential of the non-transmitting scenario depends strongly on the considered time scale. Considering a traditional O\&M time scales of 15 minutes there may not be many periods, if any, without any transmissions at all. However, LTE scheduling decisions are made per ms, i.e. per every LTE subframe; when addressing this time scale instead, the possibility for idle subframes becomes considerable, even in fairly loaded cells, something that was seen in the analysis in [8].

\section{Hardware Solutions and Radio Interface Techniques}

\subsection{Hardware Solutions}

Several hardware solutions have been defined as energy efficiency enabling techniques for base station components. Adaptability to signal level or traffic load is the key approach for energy efficient operation of base stations. This means that hardware or software solutions can decide and adjust their configuration following the traffic load variation in order to minimize the power consumption. New power-saving features take benefit by the non-uniform load distribution over the day and the short-term signal characteristics.

Investigating the base stations radio equipment aspects, a distinction is done between components for macro-cell and small-cell base stations due to the different origins and their relative weight of power consumption, c.f. Section 3.2.

The energy adaptive transmit path, defined for macro-cell base station, integrates several sub-components (see Fig.7) enabling the component deactivation and adjustment of their operating points in medium and low load situations. The digital signal processing unit (DSPU) represents the digital transceiver part and controls the energy adaptation of the other analogue transmitter components. The conversion module allows the deactivation of some of its components, controlled by the DSPU, for minimizing the power consumption when no signal is transmitted. The highest amount of power saving is achieved by the adaptive power amplifier which allows the 
adaptation and deactivation of power amplifier stages in correlation with the signal level. This is supported by the adaptive power supply by assuring the reconfiguration of supply voltages.

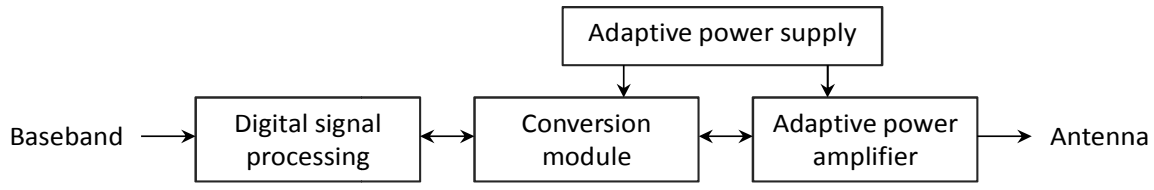

Fig. 7. Block diagram of a transmit system for macro-cell base stations

The component adaptation is strongly correlated to the signal level variation, synchronized with the LTE signal pattern. The power performance of the transmit system is defined by power characteristics showing the consumed power related to the signal load, the instantaneous level of the transmitted signal related to the maximum RF output power defined for transmission. The expected benefit is determined by comparing these characteristics with a state-of-the-art characteristic, which does not consider the proposed concepts. Measured power characteristics of the transmitter are presented in Fig.8, plotted against signal load.

Eight different operating points (OP) show a power reduction of up to $23 \%$ at low signal level, while the deactivation of components (CD) provides $55 \%$ instantaneous power savings, always compared to OP1 considered as state-of-the-art reference. The determined deactivation and reactivation transition times of $3 \mu \mathrm{s}$ respectively $10 \mu \mathrm{s}$ are short enough to allow for applying the CD feature in time slots of 2 or 3 successive LTE symbols.

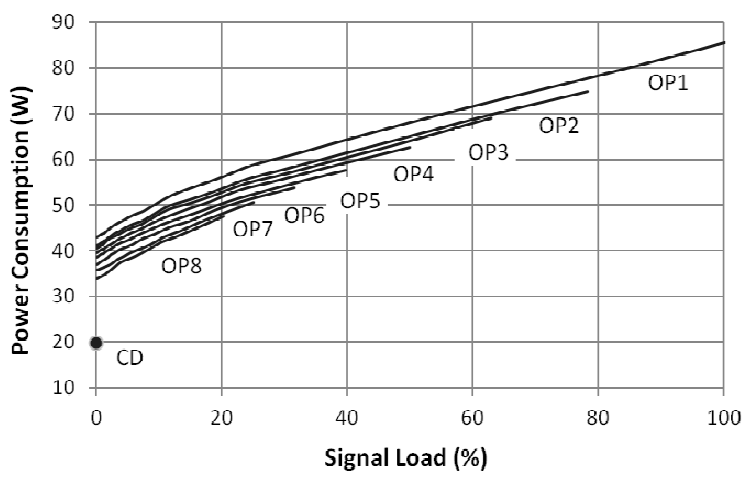

Fig. 8. Power consumption of a transmit system with $20 \mathrm{~W}$ of max. average output power

Solutions for small-cell base stations to optimize the energy efficiency or to support power management are included in all sub-components, from baseband engine to antenna interface by means of energy efficient and/or energy adaptive solutions. 
Flexible energy aware baseband signal processing algorithms are mainly beneficial in up-link at low signal loads and offer an overall power reduction of about $13 \%$ of traffic load dependent improvement. For an adaptive conversion module, flexibility of different building blocks has been introduced and lead to a traffic load dependent power efficiency improvement of $30 \%$ on average through SiNAD (signal-to-noiseand-distortion) adaptation and time/frequency duty-cycling. The reduction of power consumption depends on the signal level and shows maximum values of $58 \%$ below $5 \%$ of signal load (see Fig.9).

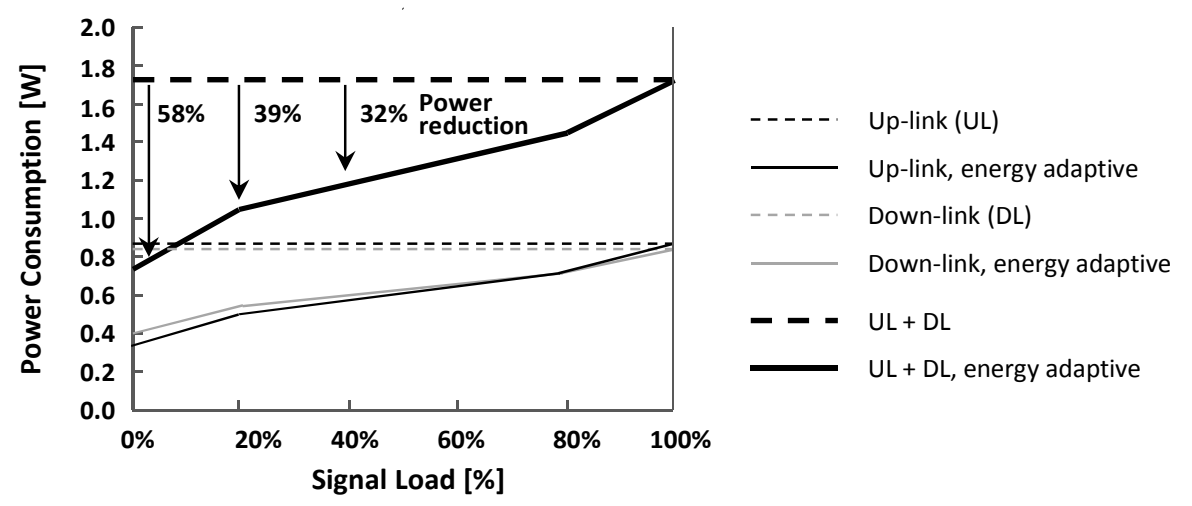

Fig. 9. Power consumption of a dual-antenna pico-cell base-station conversion module

The energy adaptive power amplifier implements the operating point adjustment and component deactivation features similar to the power amplifier of the macro-cell base station. It allow power reductions up to $55 \%$ at low signal load while up to $80 \%$ efficiency improvement can be obtained during deactivation. Such a component can be easily connected to the conversion module and is controlled via a simple interface.

\subsection{Radio Interface Techniques}

Radio interface techniques utilize the features provided by the hardware solutions in order to save energy. The benefit on energy efficiency improvement due to energy adaptive component features can be maximized by applying interface solutions acting in time and frequency domain. Duty-cycling in time groups the transmitted data over time, by maximizing the time-slots without effective transmission. It exploits the energy saving potential of hardware features which allow deactivating components in time slots of no transmission. Duty-cycling in frequency targets to reduce the spectral occupation and the resource elements and thus reduce the power of the transmitted signal. It exploits the energy saving potential of hardware features which adapt their operation for maximum energy efficiency to the level of transmitted signals.

Duty-cycling in time combined with deactivation of components enables discontinues transmission in time domain, called cell DTX. It can be realized in some different versions: 
- Micro DTX is the most straight-forward version. It exploits component deactivation during empty symbols in between transmission of cell-specific reference symbols (CRS) in the current LTE standard.

- MBSFN-based DTX is also possible within the current LTE standard. It builds on dynamic allocation of MBSFN (multicast broadcast single frequency network) subframes when possible. The energy saving potential is higher compared to micro DTX as MBSFN subframes show longer time intervals of no signal transmission.

- Short DTX assumes that no CRSs are transmitted, something that is not supported by current standards but discussed for future releases. The energy efficiency potential is higher compared to the previously mentioned methods, as deactivation can be performed during several successive sub-frames of no transmission.

- Long DTX: Exploits component deactivation during time periods of $10 \mathrm{~ms}$ or longer. It provides the highest energy efficiency potential in situations of low average traffic load.

Duty-cycling in frequency is applied in combination with the adaptation of component operation mode to the signal level, exploiting the energy efficiency potential at low and medium traffic load situations:

- Bandwidth Adaptation (BW) is based on the adjustment of the bandwidth to the required traffic load. Depending on traffic load the bandwidth can be stepwise downscaled so that lower numbers of physical resource blocks (PRBs) are allocated. Also less reference signals have to be sent.

- Capacity Adaptation (CAP) is a method, which does not change the maximum used bandwidth and the number of reference signals. An adaptation to lower load is performed by scheduling only a part of the subcarriers, i.e. limiting the number of scheduled PRBs.

Fig.10 illustrates the differences between BW Adaptation, CAP Adaptation, and Micro DTX and how they utilize the time and frequency radio resources.
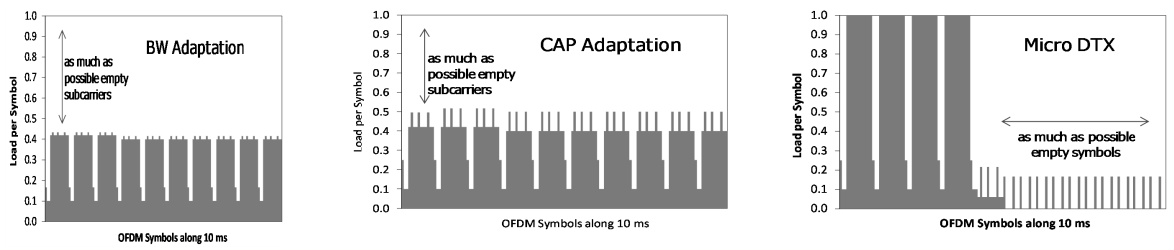

Fig. 10. Illustration of BW Adaptation, CAP adaptation, and Micro DTX

MIMO (Multiple Input Multiple Output), where several antennas are employed at transmitters and receivers, is a natural part of today's wireless systems, e.g. LTE. It is applied for maximized spectral efficiency, but leads to an energy efficiency penalty especially in low load situations. Hence, in certain situations it is beneficial from an energy efficiency point of view to down-scale to single-antenna transmission, as illustrated in Fig.11. Antenna Muting refers to fast deactivation of some antennas and the 
related transceiver components in situations of reduced traffic load. As traffic load varies quickly in a mobile broadband network, the optimization timescale of antenna muting must be in the range of milliseconds to prove beneficial. Luckily this lies within the limits of what the EARTH hardware can do. With this approach, base stations designed for highest throughput can be operated in energy efficient way also when the traffic load drops.

The energy efficient use of beamforming techniques, in which an advanced antenna is utilized to direct the transmitted signal in a narrow direction, has also been analysed by EARTH. Slow beamforming based on reconfigurable antennas, exploits medium/long term variations of traffic in order to save energy. Fast beamforming, on the contrary, is immediately following the traffic distribution and can therefore potentially save more energy. Slow beamforming based on reconfigurable antennas is also an enabler for certain network management solutions as used for Integrated Solutions.

\section{$5 \quad$ Network Level Solutions}

\subsection{Network Deployment Recommendations}

EARTH has investigated optimal cell sizes. The surprising results are somewhat counterintuitive and contradict statements found in the literature, where it often is assumed that the lower transmit power of small cells will result in smaller power consumption of the networks. However, EARTH has shown that with realistic power models reflecting state of the art of base station hardware (see Fig.5) smaller cell sizes increase the total power consumption. Therefore, traditional macro network planning, where the distance between base stations (BSs) is adjusted to the maximum inter-site distance (ISD) that provides the requested system performance and capacity, is cost efficient and also energy efficient at the same time.

For areas with ultra-high traffic demand in city hotspots, EARTH also investigated heterogeneous deployments of large macros with an underlay of small cells (heterogeneous networks). It turned out that for such localized high traffic demand, heterogeneous deployments are more efficient than a densification of the macro cell deployment. Moreover, heterogeneous deployments with femto cells are especially beneficial for indoor solutions (13\% saving). The results clearly showed that for such heterogeneous networks it is key that the macro cells can turn the offloading of their traffic into reduced energy consumption, e.g. by using adaptive EARTH BS hardware.

EARTH also studied the in practical cases very relevant scenario where operator's build on existing legacy (GSM and 3G) deployments. It turned out that a good strategy is that legacy systems will mainly provide the coverage and low-traffic demanding services in a multi-radio access technology (multi-RAT) scenario, while LTE will serve the increased capacity needs. This fact is in line with the energy consumption optimization, so the multi-RAT networks can be efficiently utilized in heterogeneous networks, as well. As a matter of fact, reality is complex so it was also identified that adopting more energy efficient RATs should be carefully balanced with the constraints coming from the forecast of capacity demand, terminal capabilities, coverage, emission limits, etc. As a relevant energy saving enabler it was estimated that site 
co-location could result in reduction in power consumption up to around 5\% due to better cooling efficiency.

Relaying, the well-known technique often used in many wireless technologies to improve data transmission at cell-edge or to provide coverage in new areas, has also energy saving merits in certain network scenarios. Relays have the potential to be energy efficient because they benefit from shorter transmission hops and from the additional receive or transmit diversity. So with efficient future relay hardware, installation of new relay nodes over a macro-only network will be more energy efficient than the deployment of additional macro nodes to serve increasing traffic demands. From the transmission point of view, several relaying techniques have been compared, and results showed that two-hop half-duplex relaying with hybrid DF/CF forwarding provides considerable gains in large macro cells. This hybrid technique is, however, not supported by current standard releases. Rooftop relays for indoor users can provide energy saving compared to macro indoor coverage.

Beyond the above described techniques focusing on densifying the network, coordination of or cooperation between BSs have been investigated as alternative solutions to cope with increased traffic by utilizing better the available bandwidth of the system. We have found that uplink CoMP is more energy efficient than noncooperative system for cell edge communication and small cell deployment. Using more than three BSs for cooperation is unlikely to be beneficial and energy efficiency can mainly be achieved via improvement in spectral efficiency as a result of macrodiversity. The most effective technology for backhaul is PON (Passive Optical Network) for today's network and AON (Active Optical Network) is a good candidate for future networks, where bandwidth requirement per BS is getting closer to Gbps.

\subsection{Network and Radio Resource Management}

The strong requirements on low latency and high system throughput result in that resources on average are not fully utilized and networks will keep using only a small fraction of their capacity [8] [14] (see Fig.6 for illustration). Therefore a key lever to obtain high savings of energy is to dynamically adapt by management the network configuration, e.g., by dynamically reducing the number of active network elements to follow adaptively the daily variation of the traffic. There is a multifold of ways to achieve such network reconfigurability for energy efficiency investigated in EARTH (see Fig.11 for illustration).

Here are some remarkable results. In urban networks, adaptive (de)sectorization of base stations can provide $30-60 \%$ energy saving without considerable impact on coverage and cell edge user throughput. Furthermore, in case of dense BS deployment, not only sectors but complete BSs can be switched off in low traffic hours providing $15-20 \%$ energy saving in single layer networks and $20-25 \%$ in vehicular scenarios. Heterogeneous networks (Hetnets) are the target of network modernization especially in densely populated urban environments as also discussed in Section 5.1. We have found that the idea of adaptive cell on/off in heterogeneous networks (even in multiRAT environment) can provide $35-40 \%$ energy saving meanwhile improving the user experience especially in indoor scenarios and in the uplink direction. 


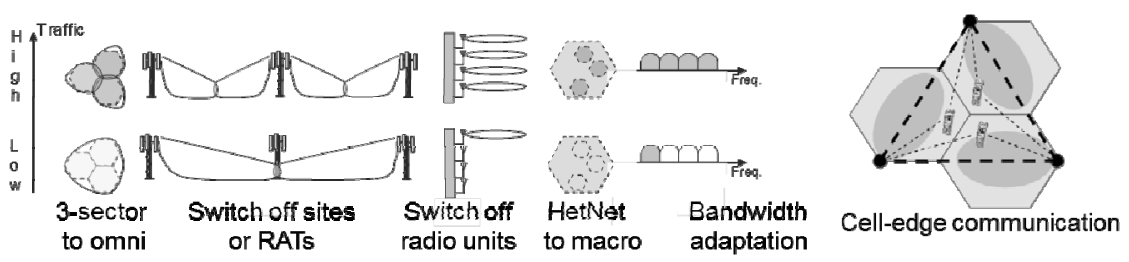

Fig. 11. Base station cooperation and traffic adaptive network reconfiguration for adaptation to traffic variation

Going further below the above timescales during the analysis of mobile systems, the goal of traditional RRM techniques should be rephrased to secure the minimal energy consumption when serving a given traffic demand with special focus on low traffic situations. RRM can support sleep modes and utilize all dimensions of RRM by finding balance between time, frequency and radiated power.

\section{$6 \quad$ Integrated Solutions}

EARTH has developed a number of different solutions for improving energy efficiency. They are ranging from base station and antenna hardware improvements, over radio interface techniques, up to solutions acting on the network level such as network management and scheduling. The solutions are developed for the 3GPP Long Term Evolution (LTE) radio technology, also known as 4G. However, some of them are general in their nature and can also be applied to other radio standards. Many of the improvements are pure implementation that can be achieved within the current standard releases, while others may need additional standard support in order to prove beneficial.

Fig.12 provides a high level overview of the relation between the different solutions. As can be seen there is an enable/utilize relation between them. At the bottom are the hardware solutions that provide certain features. The radio interface techniques in the middle level utilize these features. Finally, the network level solutions on top make use of the different radio interface techniques.

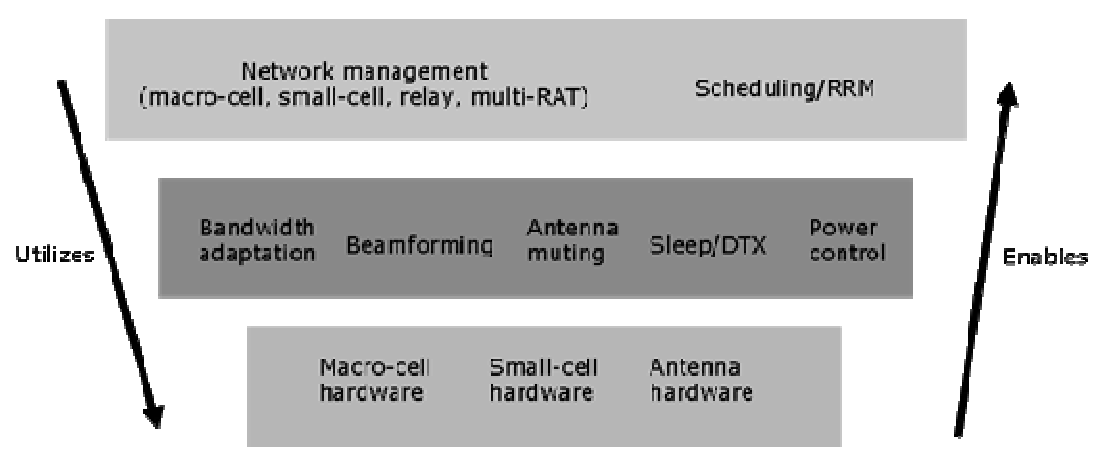

Fig. 12. Relations between EARTH solutions 
It is important to realize that the collection of solutions developed and studied in EARTH should be seen as a toolbox, and not "the" solution that should be implemented everywhere. For example, one individual solution may provide good gain in dense urban scenarios but not be attractive for the rural case. Other solutions may be suited in busy hour but detrimental during the night. Therefore, in a network, energy saving solutions should be implemented selectively.

In order to keep analysis and optimization of an entire network tractable, it is practical to consider the system to operate on different time scales, where key parameters of the network can be altered and corresponding characteristics can be changed. Typically, those changes happen on the orders of weeks \& days, hours \& minutes, and seconds \& milliseconds and energy saving solutions developed within EARTH fall into the corresponding categories Deployment \& Hardware, Network Management, and Radio Resource Allocation.

Both energy efficiency as well as energy consumption of a wireless network is governed by the strategies chosen on each time scale as depicted in Fig.13. In this regard, deployment strategies determine on the long range the number and types of equipment that are potentially active in the network. Network management techniques adapt to the daily variations of traffic and set the average activity levels of the equipment. Radio resource allocation techniques adapt network operation to variations in the channel quality as well as to small time scale variations in the traffic, in particular to idle periods of only seconds or milliseconds.

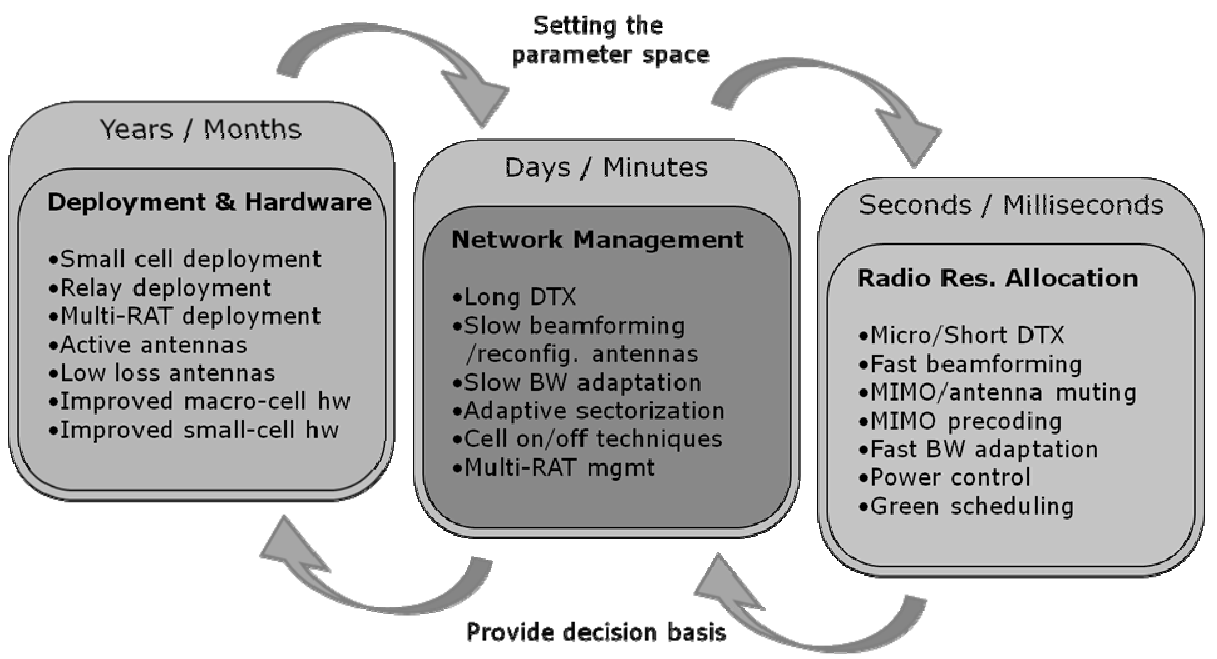

Fig. 13. Time scales of network operation

In the following, we denote by integrated solution a collection of three strategies in the area of deployment, network management, and radio resource allocation, where a strategy refers to the collection of algorithms or techniques that govern the network's characteristic behaviour on a time scale. In particular, a strategy may comprise one or more individual solutions. 
As a matter of fact, not all strategies that could be adopted on different time scales are compatible in the sense that their individual improvements in energy efficiency or energy consumption directly accumulate. As a very simple example, note that any equipment can only be sent to sleep mode once, i.e., the savings obtained from a micro sleep technique during longer idle periods can obviously not be harnessed if deep sleep mode is already activated on network management level. In general, strategies on a slower time scale define or set the parameter space and degrees of freedom for strategies on the faster scale: The deployment sets the scene for network management, whose decisions then define the degrees of freedom for resource allocation in individual cells. On the other hand, the average performance of strategies on the faster time scale is used as input or decision basis for strategies on slower time scales as depicted in Fig.13.

Based on extensive work in the project, a number of integrated solutions are defined in [15]. We will here briefly present two of them. Both solutions involve hardware solutions, radio interface techniques, as well as network level solutions. The main difference between these integrated solutions is the philosophies "deactivation" versus "adapting". The first one is deactivating sectors, antennas and timeslots when traffic can be served with less equipment running. With a very agile hardware (on PRB or OFDM symbol level) this can handle bursty traffic like file download always with full peak rate. The solution hence combines EARTH macro-cell hardware, cell micro DTX, antenna muting, and adaptive sectorization. The second philosophy is keeping the hardware running, but switches into a lower transmission mode, adapting to a lower served traffic rate. This requires less frequent switching of hardware state (e.g. on a $100 \mathrm{~ms}$ or second level). The solution involves EARTH macro-cell hardware, BW (or CAP) adaptation, and combines it with cell micro DTX.

Even though the solutions take these different philosophies, the end result is that they are able to save similar amounts of energy, approximately $70 \%$ in a country-wide LTE network according to the EARTH E ${ }^{3}$ F, see Fig. 14.

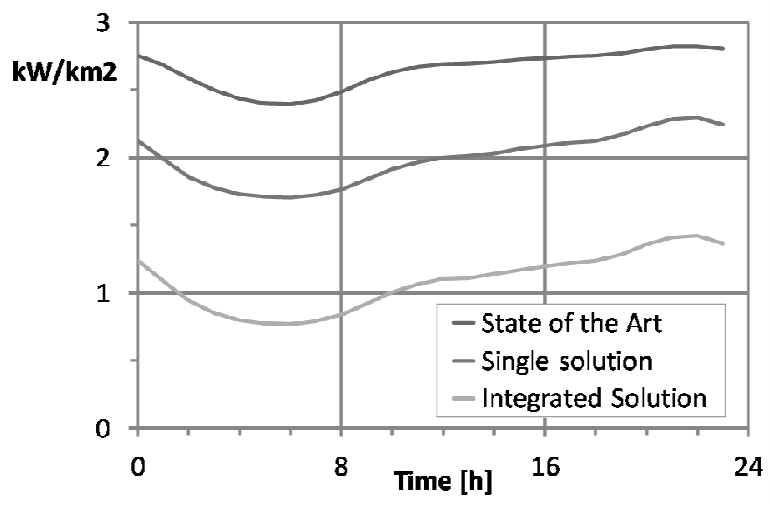

Fig. 14. Example energy saving over the day with an integrated solution 


\section{Disruptive Approaches Beyond Today's Networks}

Looking further ahead in time and beyond today's existing system standards and typical deployments, there are several promising design options and technology solutions that may enable enhanced energy efficiency in future mobile radio systems. The conclusion is that future systems / radio interfaces (beyond 2020) should be designed to minimize idle mode transmissions such as signalling, reference signals, etc. In particular, a concept where transmissions of data and system information are logically decoupled from each other (see Fig.15), can open the possibilities to more efficient sleep modes by eliminating the high signalling overhead (especially in low load) and provide $85-90 \%$ energy saving potential compared to today's systems.
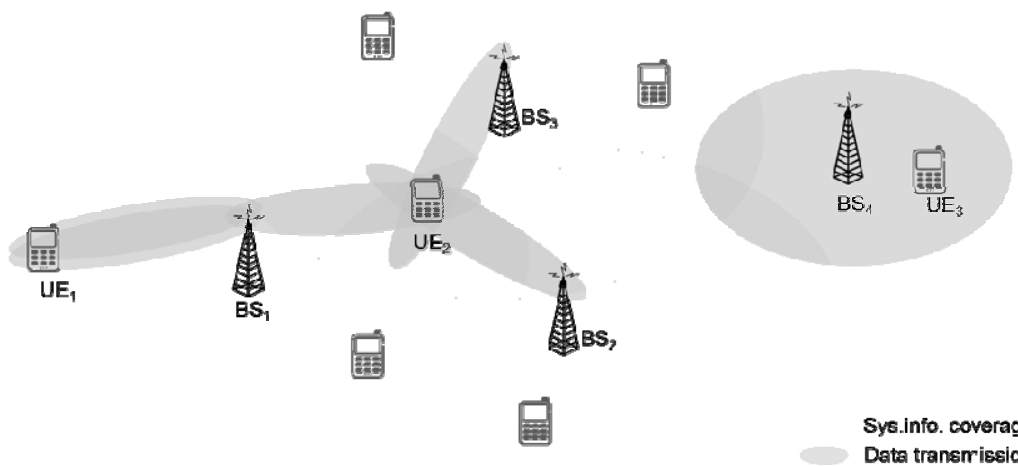

Fig. 15. Decoupling data and system information

\section{$8 \quad$ Validation of Results}

The EARTH project has realized several solutions for experimental evaluation at network deployment and management level as well as on hardware and radio interface level to demonstrate the feasibility and the proof of concepts of some key solutions in the EARTH concept. The solutions have been integrated in an operator's test plant or in hardware prototyping platforms and several measurement campaigns have been carried out in order to validate the solutions under realistic operation conditions.

Here we will briefly present these activities, while the details of the validation scenarios, setups and validation test results are found in [16].

\subsection{Validation of Hardware Concepts by Transceiver Prototypes}

The novel hardware solutions developed by EARTH are key enablers for many of the other EARTH concepts for energy efficient mobile systems. These hardware solutions have been realized for macro-cell and pico-cell transceivers and have been evaluated 
by measurements. Examples of these prototypes are shown in Fig.16. The obtained power characteristics (see Section 4.1) delivered the instantaneous behaviour for different signal levels and allowed the validation of the base station power models used in the project for system level simulations. By means of realistic LTE signals, the dynamic performance of the hardware components could be studied. The transition times during the deactivation of different components with maximum $10 \mu$ s for high power amplifiers and much lower for components operating at lower power level, demonstrated that component deactivation can be applied for LTE signals even in short time slots of 2 or 3 successive symbols. This has been validated even with regard to the spectral signal performance by a successful transmission of LTE signals when operating the component adaptation or deactivation features.

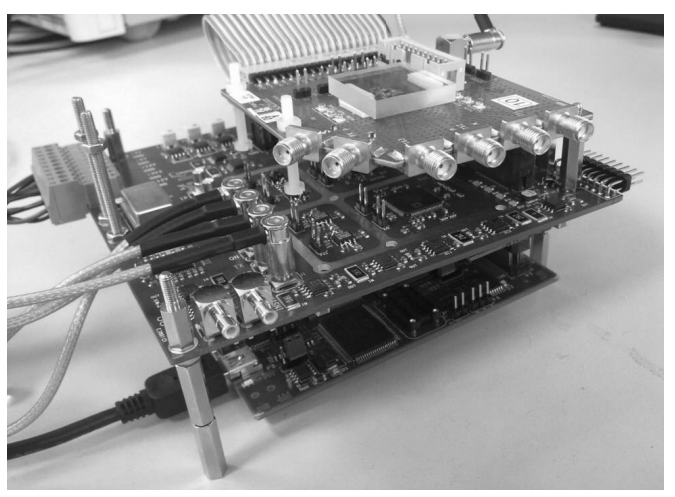

(a) Adaptive small signal transceiver

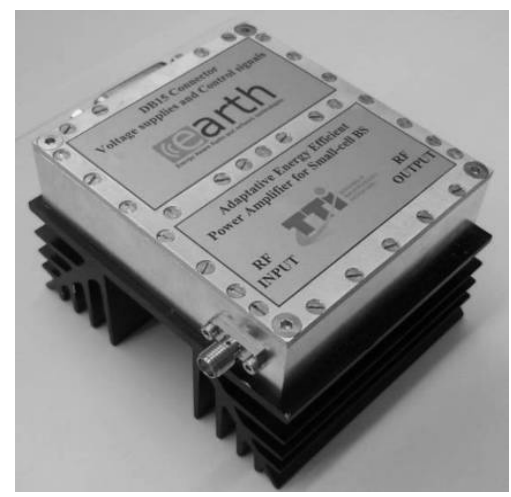

(b) Adaptive power amplifier

Fig. 16. Pico-cell transceiver prototype components

\subsection{Validation of Network Level Solutions in an Operator Test Plant}

One of the key solutions of radio access network management proposed by the EARTH project is the possibility to switch a cell on and off in order to adapt the network configuration to traffic demand. Therefore it was identified as important to validate and demonstrate the feasibility to do this. The cell on/off scheme is mainly based on a network management software tool designed and developed by EARTH to reduce the network power consumption at all the levels of traffic loads, with a proper focus on low traffic loads. Its main objective is to reduce the power consumption, on the basis of the number of users registered and attached inside an area of radio coverage and of the corresponding traffic demands, by switching off and on a single cell. Energy savings are possible by redistribution of the users over the cells of a base station and by enabling to switch off cells that are not serving users. The cell on/off scheme has been implemented and validated by setting up in Telecom Italia test plant a proper test scenario and by performing real measurements with the cell on/off scheme in operation.

The experimental study carried out has shown that it is feasible to implement such a scheme on a commercial base station, and that energy savings indeed are possible. 
The experimental studies carried out have shown that already the application of a specific EARTH network management solution in a network made up by commercial base station allows for daily savings in the order of $15 \%$. This is very well in line with the savings predicted by simulations and confirms that integrating such solution with the EARTH hardware and the other EARTH solutions has really the potential to provide the $>50 \%$ savings as predicted by simulations (see Section 6 ).

\section{Summary and Conclusion}

The EARTH project had the ambition to pioneer the research on sustainability and energy efficiency of mobile broadband. Indicators that EARTH was successful in this are listed in the following bullet points:

- EARTH developed a methodology, $\mathrm{E}^{3} \mathrm{~F}$, for assessment of RAN energy consumption and energy efficiency. The methodology has been adopted also outside the project in other research initiatives and provides foundations in standardization towards characterizing network energy efficiency, e.g., in ETSI Eco-environmental Product Standards.

- EARTH developed key solutions for improved energy efficiency of such infrastructure. It found ways to integrate hardware, deployment and management solutions efficiently into an Integrated Solution that allows decreasing energy consumption by more than $50 \%$.

- EARTH implemented key constituents of its solutions in hardware and software prototypes, illustrating the feasibility and proving validity of the developed novel solutions and of their foreseen savings in an operator's testbed under realistic operation conditions. So EARTH ensured that its theoretical savings will be also practical savings.

- EARTH analysed for the first time the impact of Future Internet on sustainability and energy demands of mobile communications infrastructure. It showed that the EARTH Integrated Solution allows avoiding an increase of $\mathrm{CO}_{2} \mathrm{e}$ emissions and energy demands whilst expanding the mobile infrastructure to satisfy the future traffic demands. EARTH results are therefore pivotal for a sustainable and environment friendly growth of mobile broadband communications as needed to bridge the digital divide and allowing for smart growth enabled by mobile infrastructure.

The EARTH project was committed to have a high impact. Fig.17 depicts how the EARTH results bring about impact in the different areas.

Furthermore, EARTH also had impact in standards and in the scientific community as well as among the general public. For example, the EARTH white paper "Challenges and Enabling Technologies for Energy Aware Mobile Radio Networks" published in IEEE communication Magazine [17] was in the top ten list of papers downloaded in November 2010 [18]. EARTH also was awarded the 4th Future Internet Award at the Aalborg's edition of FIA in 2012 [19], for its enabling contributions to sustainable and environment friendly growth of mobile broadband infrastructure, bridging the digital divide and supporting smart growth. The European Commission 
Vice President Neelie Kroes commented: "The ICT sector is growing but its carbon footprint should not follow. I congratulate the partners of the EARTH project who have found ways to deliver the services we need while reducing $\mathrm{CO}_{2}$ emissions and cutting down on energy bills." [20].

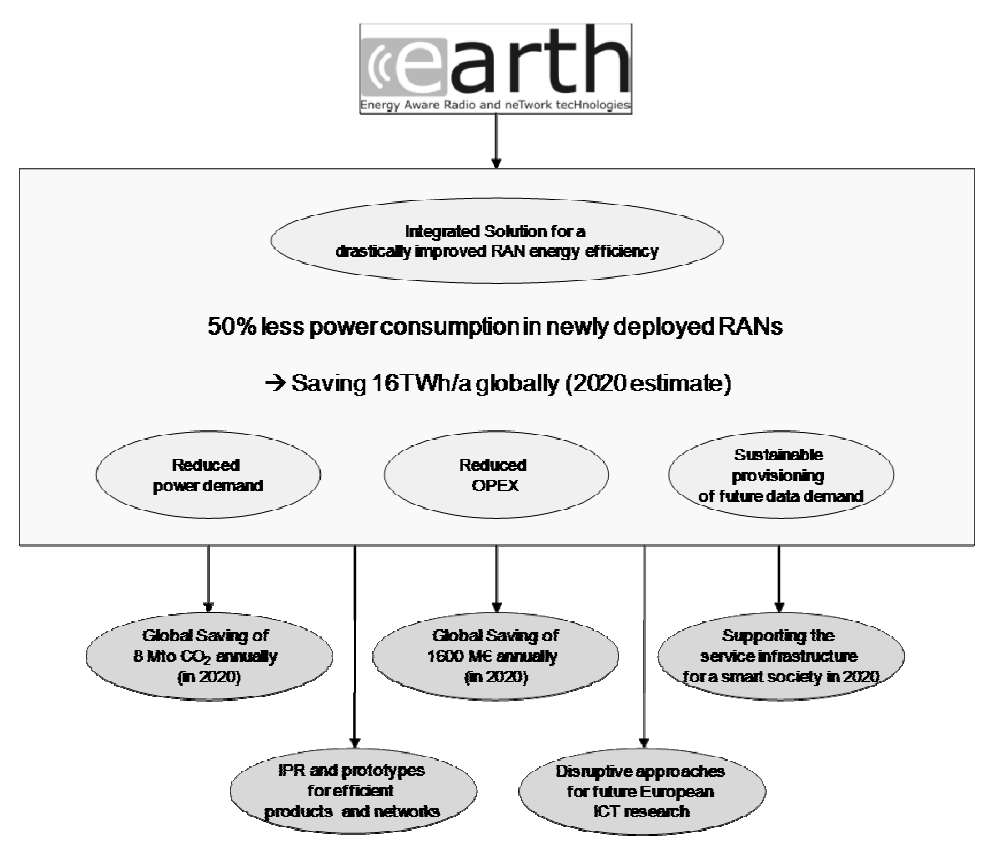

Fig. 17. EARTH results and the resulting savings bring about their socio-economic impact

For further information and details on EARTH, we refer to the comprehensive public website (https://www.ict-earth.eu/) which contains all relevant information about the project, such as the project vision and objectives, the relation of the project to the funding program and other projects in the same domain and the EARTH consortium details. Also the public deliverables of the consortium are available for download at this site.

Acknowledgements. The research leading to these results has received funding from the European Community's Seventh Framework Programme FP7/2007-2013 under grant agreement $\mathrm{n}^{\circ} 247733$ - project EARTH.

The authors would like to thank all the EARTH partners for the fruitful joint work leading to the results presented in this chapter.

Open Access. This article is distributed under the terms of the Creative Commons Attribution Noncommercial License which permits any noncommercial use, distribution, and reproduction in any medium, provided the original author(s) and source are credited. 


\section{References}

1. http://www.ict-earth.eu

2. The Europe 2020 strategy, http://ec.europa.eu/europe2020/europe-2020-in-anutshell/priorities/index_en.html

3. SMART2020 report with respect to footprint of mobile networks. Technical report, GeSI Global e-Sustainability Initiative, http://www.gesi.org/Reports Publications / Smart2020/tabid/192/Default.aspx

4. EU Commissioner Calls on ICT Industry to Reduce Its Carbon Footprint by $20 \%$ as Early as 2015. Press release, MEMO/09/140 (2009)

5. Fehske, A., Fettweis, G., Malmodin, J., Biczók, G.: The Global Footprint of Mobile Communications: The Ecological and Economic Perspective. IEEE Communications Magazine 49(8), 55-62 (2011)

6. Energy Efficiency of Wireless Access Network Equipment. Technical specification, ETSI Environmental Engineering (EE), ETSI TS 102706 V1.2.1 (2011), http://www.etsi.org/deliver/etsi_ts/102700_102799/102706/

7. An Energy Efficiency Benchmarking Service for Mobile Network Operators. Technical report, GSMA (2011), http: / / www. gsmworld. com/mee

8. Auer, G., Vito, G., Desset, C., Gódor, I., Skillermark, P., Olsson, M., Imran, M.A., Sabella, D., Gonzalez, M.J., Blume, O., Fehske, A.: How much Energy is needed to run a Wireless Network? IEEE Wireless Communications 18(5), 40-49 (2011)

9. Environmental Engineering (EE); Principles for Mobile Network level energy efficiency. Technical report, ETSI TR103117

10. Green Touch Initiative, Mobile Communications Working Group, http://www.green touch. org/index.php?page=mobile-communications-working-group

11. Desset, C., Debaillie, B., Giannini, V., Fehske, A., Auer, G., Holtkamp, H., Wajda, W., Sabella, D., Richter, F., Gonzalez, M.J., Klessig, H., Gódor, I., Olsson, M., Imran, M.A., Ambrosy, A., Blume, O.: Flexible power modelling of LTE base stations. In: IEEE WCNC 2012, Paris, pp. 2858-2862 (2012)

12. Base station power model. Technical report, 3GPP TSG-RAN WG1 \#67, R1-114336, DOCOMO, Ericsson, Alcatel-Lucent, Telecom Italia

13. http: //www . umts-forum. org

14. Mobile internet phenomena report. Technical report, Sandvine (2010), http: / / www . sandvine. com/downloads / documents / $2010 \% 20 \mathrm{Global} \% 20$ Internet\%20Phenomena\%20Report.pdf

15. Olsson, M. (ed.): Deliverable 6.4: Final Integrated Concept. Technical report, INFSO-ICT247733 EARTH (Energy Aware Radio and NeTworkTecHnologies) (2012)

16. Tomaselli, W. (ed.): Deliverable 5.3: Report on Validation. Technical report INFSO-ICT247733 EARTH (Energy Aware Radio and NeTworkTecHnologies) (2012)

17. Correia, L., Zeller, D., Blume, O., Ferling, D., Jading, Y., Gódor, I., Auer, G., Van Der Perre, L.: Challenges and Enabling Technologies for Energy Aware Mobile Radio Networks. IEEE Communications Magazine 48(11), 66-72 (2010)

18. http: / /www. comsoc.org/topten/november-2010-top-ten

19. Earth Project Wins Aalborg Future Internet Competition (May 2012), http: / /www.cefims.eu/2012/05/earth-project-wins-aalborgfuture-internet-competition/

20. Digital Agenda: EU research breakthrough will cut 4G / LTE mobile network energy use in half. Press release, EC, http://europa.eu/rapid/press-release_MEMO12-327_en.html 